\title{
CARACTERISTICAS DE IDENTIDADE, QUALIDADE E ESTABILIDADE DA MANTEIGA DE GARRAFA. PARTE II - ESTABILIDADE ${ }^{1}$
}

\author{
Carmem Lygia Burgos AMBRÓSIO ${ }^{2}$, Nonete Barbosa GUERRA ${ }^{2, *}$, Jorge MANCINI FILHO ${ }^{3}$
}

\begin{abstract}
RESUMO
Com vistas a estabelecer o tempo de vida útil da manteiga de garrafa, duas marcas deste produto (A e B) de ampla comercialização na cidade do Recife foram avaliadas quanto a estabilidade durante o armazenamento a $25^{\circ} \mathrm{C}$ a intervalos de $0,30,60,90$ e 120 dias através da determinação do indice de peróxido, acidez, análise cromatográfica de ácidos graxos e análise sensorial. Segundo os resultados, a acidez apresentou uma elevação acentuada nos primeiros 30 dias de armazenamento estabilizando-se a seguir até 120 dias. O índice de peróxido aumentou ao longo do tempo sendo acompanhado pela intensificação do "flavour" de ranço para ambas amostras que a partir dos 90 dias de armazenamento foi referido como extremamente forte por $50 \%$ dos provadores. Quanto aos ácidos graxos, foi constatada diminuição do percentual do linoléico (18:2) na manteiga A embora nenhuma mudança tenha ocorrido no conteúdo de ácidos graxos trans para as duas manteigas. Os resultados demonstram que o tempo de armazenamento não exerce influência significativa e que a manteiga de garrafa apresenta uma curta vida-de-prateleira face a oxidação lipídica que a torna inadequada para consumo após 60 dias a partir da data de fabricação.

Palavras-chave: manteiga; estabilidade; vida-de-prateleira; identidade; qualidade.
\end{abstract}

\section{SUMMARY}

THE STABILITY OF A BRAZILIAN REGIONAL BUTTER "Manteiga de Garrafa". Trying to establish the time of shelf-life of "manteiga de garrafa" (a kind of regional Brazilian butter), two brands of this product (A and B) of wide commercialization in the city of Recife were analyzed as the stability during the storage at $25^{\circ} \mathrm{C}$ to intervals of $0,30,60,90$ and 120 days through the determination of the peroxide value, $\%$ free fattty acid, fatty acid composition and sensorial evaluation. According to the results, acidity increased in the first 30 days of storage being stabilized up to 120 days, the peroxide value increased along the time being accompanied by the intensification of the "flavour" of rancidity for both samples that it was referred as extremely strong for $50 \%$ of the panelists starting from the 90 days of storage; with relationship to the fatty acids, decrease of the percentile of the linoleic acid (18:2) was verified in the brand A although no change has occurred in the content of trans-fatty acid in the two brands. The results has demonstrated that the time of storage doesn't show significant influence and this product presents a short shelf-life because of lipid oxidation that turns it inadequate for consumption 60 days after production date.

Keywords: butter; stability; shelf-life; identity; quality.

\section{1 - INTRODUÇÃO}

A maior parte da manteiga de garrafa comercializada no Nordeste não apresenta rótulo. Na cidade de Recife apenas duas marcas possuem rotulagem, na qual se encontra especificado local de produção, registro de inspeção estadual, data de fabricação e, inclusive, validade, sem que se tenha conhecimento da realização de estudos de vida-de-prateleira para sua determinação.

A manteiga de garrafa é uma gordura anidra e, portanto, bastante susceptivel a oxidação lipídica, cujo processo de fabricação é artesanal sem adequado controle das etapas de processamento.

Durante o processamento, estocagem e utilização dos óleos e gorduras, reações de hidrólise e oxidação podem desencadear características indesejáveis aos produtos. Tais características englobam redução do valor nutritivo e desenvolvimento de sabores e odores

Recebido para publicação em 08/08/2001. Aceito para publicação em 22/07/2003 (000721). Parte da dissertação de mestrado de C.L.B. Ambrósio.

${ }^{2}$ Departamento de Nutrição - Universidade Federal de Pernambuco (UFPE)

- Campus Universitário - CEP: 50670-901/Tel: (81) 32718470/Fax: (81) 32718473/http://recife nutricao ufpe. br.

${ }^{3}$ Departamento de Alimentos e Nutrição Experimental - Faculdade de Ciências Farmacêuticas - Universidade de São Paulo (USP).

* A quem a correspondência deve ser enviada. desagradáveis ou de "ranço", que reduzem o tempo de prateleira dos alimentos, tornando-os inaceitáveis pelos consumidores $[1,5,16]$.

A literatura referente a manteiga de garrafa é bastante escassa e, em especial, quanto aos efeitos das condições de armazenamento que é restrita a abordagem realizada por SANTOS [10] do ponto de vista biológico, cujos resultados levaram o autor a concluir que a estabilidade química da manteiga de garrafa é afetada pela elevação da temperatura, exposição à luz e à umidade, acarretando prejuízos significativos em sua qualidade nutricional.

Como até o presente não foram encontrados registros sobre a estabilidade da manteiga de garrafa ao longo do tempo de armazenamento, e sendo esta uma exigência da atualidade, constata-se a importância desta pesquisa voltada para o estudo da vida-de-prateleira do produto com vistas a garantir a saúde do consumidor.

\section{2 - MATERIAL E MÉTODOS}

\section{1 - Obtenção e tratamento das amostras}

Foram utilizadas nesta pesquisa amostras de duas marcas de manteiga de garrafa, acondicionadas em garrafas de vidro transparente (originais) com capacidade para $200 \mathrm{~mL}$, adquiridas em dois hipermercados da cidade do Recife nas condições de comercialização: tem- 
peratura de aproximadamente $25^{\circ} \mathrm{C}$ e presença de luz artificial.

Na seleção das amostras foram adotados os seguintes critérios: presença de rótulo com registro de inspeção estadual, pertencer ao mesmo lote e apresentar um tempo de prateleira de, no máximo, dois dias a partir da data de fabricação.

De cada marca de manteiga foram coletadas, aleatoriamente, 11 unidades que foram distribuidas em 2 lotes e tratadas conforme se segue:

- Lote 1 - Constituído por 6 unidades, que foram armazenadas em condições similares àquelas observadas nos hipermercados nas embalagens originais e a intervalos de 0, 30, 60, 90 e 120 dias foram tomadas aliquotas de cada recipiente para avaliação da estabilidade da manteiga de garrafa ao longo do tempo de armazenamento.

- Lote 2 - Composto por 5 amostras, foi submetido às condições de armazenamento anteriormente descritas e destinado à análise sensorial.

\section{2 - Métodos analíticos}

A estabilidade da manteiga de garrafa foi avaliada através das seguintes determinações; de acordo com as técnicas descritas nas "Normas Analiticas do Instituto Adolfo Lutz" [7]:

- Índice de peróxido (mEq/kg)

- \% de Ácidos graxos livres (oléico)

- Análise cromatográfica de ácidos graxos

A análise cromatográfica foi realizada pelo Laboratório de Lipides no Departamento de Alimentos e Nutrição Experimental da Faculdade de Ciências Farmacêuticas - Universidade de São Paulo (USP).

A esterificação dos ácidos graxos foi efetuada de acordo com a técnica de HARTMAN \& LAGO [6].

Para determinação dos ácidos graxos foi utilizado um cromatógrafo a gás HP 6890 com coluna cromatográfica (marca SUPELCO) de silica fundida SP-2560

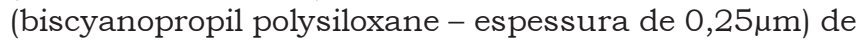
$100 \mathrm{~m}$ de comprimento e $0,25 \mathrm{~mm}$ de diâmetro interno nas seguintes condições: programação de temperatura da coluna a $1^{\circ} \mathrm{C} / \mathrm{min}$ de $125^{\circ} \mathrm{C}$ a $175^{\circ} \mathrm{C}$, permanecendo nesta temperatura por 20 minutos; temperatura do injetor: $225^{\circ} \mathrm{C}$; temperatura do detector: $235^{\circ} \mathrm{C}$; gás de arraste: hélio $92 \mathrm{~mL} / \mathrm{min}$, razão de divisão da amostra no injetor: $1 / 50$.

- Análise sensorial

Foi utilizado o teste QDA conforme STONE \& SIDEL [13]. As amostras foram avaliadas quanto ao desenvolvimento do "flavour" de ranço ao longo do tempo de armazenamento, através de um painel sensorial constituído por 7 provadores treinados. Foi utilizada escala hedônica estruturada de 9 pontos na qual a nota 0 (zero) correspondia a ausência de "flavour" de ranço e nota 9 (nove) "flavour" de ranço extremamente forte. As amostras foram servidas com torradas e água.

\section{3 - Análise estatística}

Os dados obtidos foram submetidos ao teste de Duncan, ao nivel de 5\% de significância, para comparação das médias das amostras A e B ao longo do tempo de armazenamento e correlação de Pearson visando a correlação dos atributos de qualidade mais relevantes.

A análise estatística foi realizada através do programa "Statistica for Windows", STATSOFT [12].

\section{3 - RESULTADOS E DISCUSSÃO}

Durante quatro meses de armazenamento, as duas marcas em estudo foram monitoradas quanto à acidez, indice de peróxido, avaliação do "flavour" de ranço e composição de ácidos graxos, com vistas a obter dados que permitissem estabelecer o prazo de validade das mesmas.

De acordo com as Figuras 1, 2 e 3, constata-se uma evolução da reação de oxidação em função do tempo. A análise acurada da Figura 1 demonstra uma elevação significativa da acidez entre 0 e 30 dias, equivalente a 2 e 2,5 vezes o valor inicial para a manteiga $A$ e $B$ respectivamente, seguida de um patamar até 120 dias. Verifica-se ainda que, embora tenha sido mantida uma diferença significativa entre as marcas, ambas apresentaram uma mesma tendência até o final do experimento.

Em relação ao indice de peróxido (Figura 2), os resultados foram distintos para as duas marcas. Enquanto a manteiga A apresentou uma evolução linear ao longo do tempo de armazenamento, a marca B, com o menor indice de peróxido inicial, ultrapassou-a entre 60 e 90 dias, mantendo-se estável até 120 dias. A diferença registrada entre os valores do tempo inicial e final do experimento indica uma maior velocidade de propagação da reação de oxidação na manteiga $B$. Ao final do estudo, ambas as marcas apresentaram indices de peróxido superiores aos relatados por VIEIRA [14] para manteiga e por VYAS \& VYAS [15] para o "butteroil" e inferiores aos obtidos por ISKANDER, BAYOUMI \& SHALABI [8] para o "ghee" (produto similar indiano).

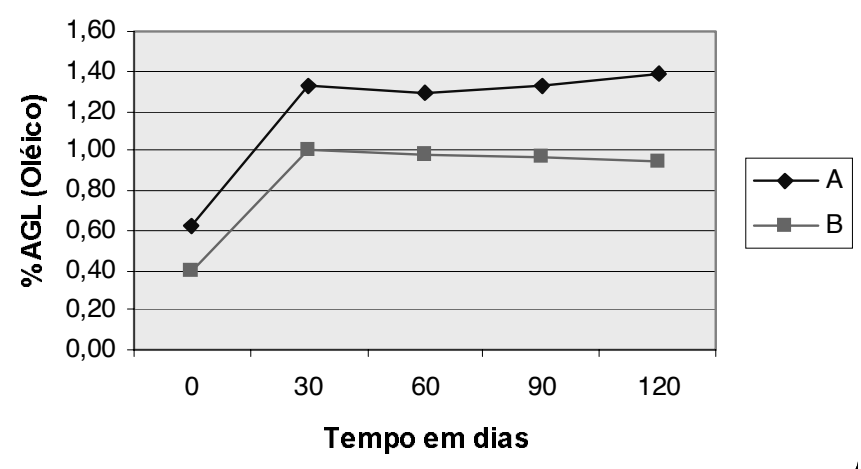

FIGURA 1. Variação percentual de ácidos graxos livres - \%AGL (Oléico) das manteigas de garrafa A e B ao longo do tempo de armazenamento. 


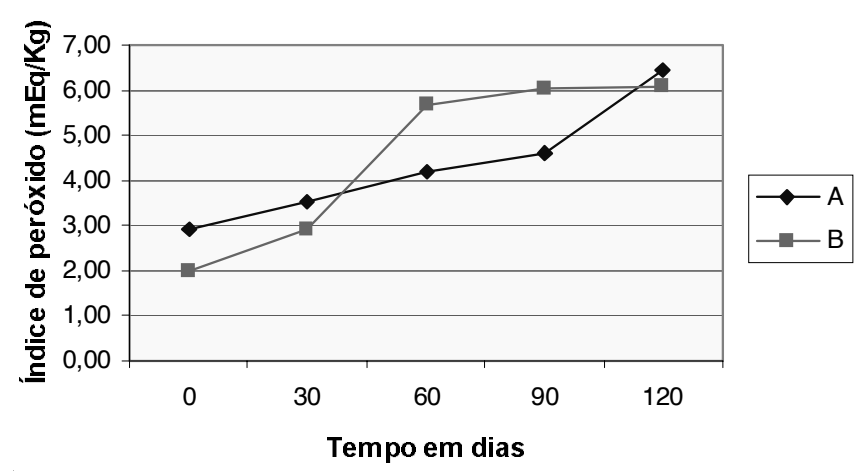

FIGURA 2. Variação do índice de peróxido (mEq/Kg) das manteigas de garrafa A e B ao longo do tempo de armazenamento.

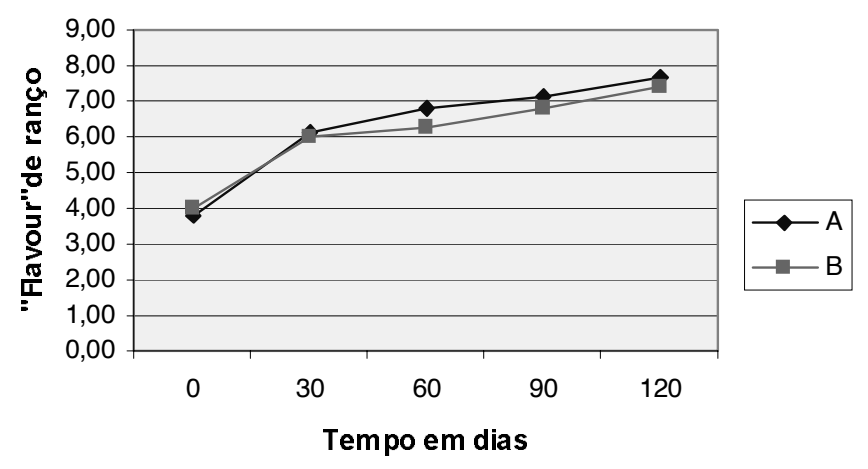

FIGURA 3. Variação das notas (médias) de "flavour" de ranço das manteigas de garrafa A e B ao longo do tempo de armazenamento atribuídas pelos 7 provadores treinados.

A maior estabilidade apresentada pelo "butteroil" justifica-se provavelmente pelo emprego de antioxidantes na sua elaboração. A este respeito, a legislação vigente no país, Portaria no 146 do Ministério da Agricultura [4] permite a utilização desses compostos no processamento do "butteroil" não destinado a elaboração de produtos lácteos. Pesquisas também vêm sendo implementadas com vistas a controlar a oxidação lipídica do "ghee" procedimento que poderia ser estendido à manteiga de garrafa [3, 9].

A avaliação sensorial do "flavour" de ranço (Figura 3) ratificou os resultados obtidos através da determinação dos parâmetros quimicos anteriormente descritos. Entretanto, convém ressaltar que após 90 dias de armazenamento, mais de 50\% dos provadores referiram o "flavour" ranço como extremamente forte para o consumo.

Ao correlacionar o "flavour" de ranço ao longo do tempo com o indice de peróxido e acidez foi constatado que o coeficiente de correlação entre a marca A e os parâmetros químicos: indice de peróxido $(\mathrm{r}=0,73)$ e acidez $(\mathrm{r}=0,83)$, foram mais elevados que os determinados para a marca $B$ $(r=0,67)$ e $(r=0,60)$, respectivamente. Estes resultados, somados aos anteriormente apresentados, confirmam as diferenças registradas quanto aos aspectos de qualidade entre as duas marcas durante a realização da pesquisa.
A análise da composição de ácidos graxos (Tabela 1) permite constatar que não houve elevação do percentual dos ácidos graxos trans-isômeros ao longo dos 4 meses, fato que contradiz SMITH et al. [11] ao sugerirem que a oxidação, durante o armazenamento de alimentos, pode aumentar o conteúdo desses compostos. Quanto aos demais ácidos graxos, apenas o percentual de ácido linoléico (18:2) na manteiga A apresentou uma redução gradativa com o tempo, provavelmente dada a sua susceptibilidade à oxidação que nesta marca foi mais elevada nos momentos iniciais.

TABELA 1. Composição de ácidos graxos (em área \%) das manteigas de garrafa A e B ao longo do tempo de armazenamento.

\begin{tabular}{|c|c|c|c|c|c|c|c|c|}
\hline \multirow{4}{*}{ Ácidos Graxos } & \multicolumn{8}{|c|}{ Tempo de Armazenamento } \\
\hline & \multicolumn{2}{|c|}{ Tempo Zero } & \multicolumn{2}{|c|}{30 Dias } & \multicolumn{2}{|c|}{90 Dias } & \multicolumn{2}{|c|}{120 Dias } \\
\hline & Manteiga & Manteiga & Manteiga & Manteiga & a Manteiga & Manteiga & Manteiga & a Manteiga \\
\hline & A & B & $\mathrm{A}$ & B & A & B & $\mathrm{A}$ & B \\
\hline Caprîico (8:0) & 1,09 & 1,15 & 1,03 & 1,07 & 1,60 & 1,11 & 1,12 & 1,16 \\
\hline Cáprico (10:0) & 2,94 & 2,71 & 2,70 & 2,79 & 3,61 & 2,57 & 2,56 & 2,73 \\
\hline Láurico (12:0) & 3,44 & 3,15 & 3,18 & 3,29 & 4,16 & 3,02 & 3,00 & 3,18 \\
\hline Mirístico (14:0) & 11,48 & 10,98 & 10,93 & 11,22 & 12,40 & 10,72 & 10,94 & 11,13 \\
\hline Miristoléico (14:1) & 0,97 & 1,06 & 0,91 & 0,95 & 1,33 & 1,03 & 0,84 & 0,91 \\
\hline Pentadecanóico (15:0) & 1,40 & 1,55 & 1,29 & 1,39 & 1,59 & 1,47 & 1,25 & 1,29 \\
\hline Palmítico (16:0) & 31,07 & 31,39 & 28,46 & 31,14 & 36,35 & 31,07 & 29,90 & 31,42 \\
\hline Palmitoléico (16:1) & 1,31 & 1,44 & 1,32 & 1,31 & 1,75 & 1,38 & 1,19 & 1,27 \\
\hline Margárico (17:0) & 0,87 & 0,91 & 0,87 & 0,84 & 0,78 & 0,94 & 0,66 & 0,82 \\
\hline Heptadecenóico (17:1) & 0,27 & 0,33 & 0,27 & 0,27 & 0,32 & 0,31 & 0,20 & 0,26 \\
\hline Esteárico (18:0) & 11,88 & 11,62 & 12,92 & 12,07 & 8,68 & 11,32 & 13,49 & 11,99 \\
\hline $18: 1(6-8 t+9 t+10 t)$ & 3,71 & 3,69 & 3,95 & 3,89 & 1,20 & 3,31 & 2,34 & 2,30 \\
\hline Oléico (18:1) & 21,32 & 22,46 & 24,53 & 20,82 & 17,66 & 21,69 & 23,41 & 20,62 \\
\hline $18: 2(9 c+10 c+11 c+13 c)$ & - & - & 0,41 & - & - & - & - & - \\
\hline Linoléico (18:2) & 3,71 & 2,60 & 2,15 & 3,73 & 1,85 & 2,51 & 1,78 & 3,68 \\
\hline Linolênico (18:3) & 0,48 & 0,60 & 1,00 & 0,49 & 0,81 & 0,53 & 0,82 & 0,41 \\
\hline Eicosanóico (20:0) & - & 0,09 & - & 0,08 & - & - & - & - \\
\hline Não identificados & 4,07 & 4,32 & 4,14 & 3,90 & 6,44 & 6,90 & 6,52 & 6,64 \\
\hline
\end{tabular}

A peroxidação dos ácidos graxos polinsaturados leva a formação de malonaldeído, que pode provocar ligações cruzadas nas lipoproteínas LDL levando ao acúmulo de colesterol nos vasos sangüineos, e conseqüentemente a problemas cardiovasculares e cancerígenos [2]. Tais afirmações são motivos de preocupação, uma vez que de acordo com os resultados obtidos no presente estudo, as manteigas de garrafa analisadas já apresentavam um nível considerável de oxidação no periodo inicial de armazenamento, em especial, no que diz respeito ao indice de peróxido.

Estas constatações demonstram a necessidade de intervir no processo de obtenção da manteiga de garrafa, de modo a manter a oxidação dos lipídios dentro dos padrões permitidos pela legislação em vigor para produtos similares.

\section{4 - CONCLUSÕES}

Os resultados obtidos, nas condições em que foi realizada a presente pesquisa, permitem concluir que: 
- A manteiga de garrafa é um produto altamente susceptivel à oxidação lipídica.

- O reduzido conteúdo de ácidos graxos trans demonstra que o tempo de armazenamento não exerce influência na formação destes compostos.

- O produto apresenta uma curta vida-de-prateleira face à oxidação lipídica que o torna inadequado para consumo após 60 dias, a partir da data de fabricação.

\section{5 -REFERÊNCIAS BIBLIOGRÁFICAS}

[1] AKOH, C. C., MIN, D. B. Food Lipids : chemistry, nutition, and biotechnology. New York: Marcel Dekker, 1998. 816p.

[2] ARAÚJO, J. M. A. Química de alimentos: teoria e prática. Viçosa : Imprensa Universitária da Universidade Federal de Viçosa, 1995. 332p.

[3] BINDAL, M. P. , WADHWA, B. K. Renovation of rancid ghee. Indian Journal of Dairy Science, v. 44, n. 5, p. 323326, 1991.

[4] BRASIL. Ministério da Agricultura. Portaria n. ${ }^{\circ} 146$ de 07 de mar. 1996. Diário Oficial da União, Brasília, 11 de março de 1996.

[5] FEnNEMA, O. R. Química de los alimentos. Zaragoza : Acribia, 1992. 1095 p.

[6] HARTMAN,L., LAGO, R.C. A . Rapid preparation of fatty acid methyl ester from lipids. Londres : Lab. Pract., v. 22, p. 475-476, 1973.

[7] INSTITUTO ADOLFO LUTZ. Normas Analíticas do Instituto Adolfo Lutz : métodos químicos e físicos para análise de alimentos. 3.ed. São Paulo, 1985. v. 1, $533 \mathrm{p}$.
[8] ISKANDER, M.H., BAYOUMI. S. E., SHALABI, S.I. Composition and storage stability of commercial anhydrous milk fat and hydrogenated oils. Journal of Food Technology, v. 20, p. 83-88, 1985.

[9] PARMAR, S. S., SHARMA, R. S. Effect of mango (Mangifera indica L.) seed kernels pre-extract on the oxidative stability of ghee. Food Chemistry, v. 35, p. $99-107,1990$.

[10] SANTOS, E. P. Estabilidade química da manteiga da terra. Bananeiras : Universidade Federal da Paraíba, 1995. 84p. Dissertação. Mestrado. Departamento de Tecnologia Rural - UFPB.

[11] SMITH, L. M., DUNKLEY, W. L., FRANKE, A., DAIRIKI, T. Measurement of trans and other isomeric unsaturated fatty acids in butter and margarine. Journal of the American Oil Chemists Society, v. 55, p. 257-261, 1978.

[12] STATSOFT, Inc. Statistica for Windows ? Computer program manual?. Tulsa, OK : StatSoft, Inc., 1996.

[13] STONE, H.,SIDEL,J.L. Sensorial evalution practices. Orlando: Academic Press, 1985. 311p.

[14] VIEIRA, M. L. M. Oxidação lipídica em manteiga: avaliação de métodos analíticos. Recife : Universidade Federal de Pernambuco, 1994. 84p. Dissertação Mestrado. Departamento de Nutrição.

[15] VYAS, M. N. , VYAS, S.H. Keeping quality of commercial butteroil stored at different storage temperatures. Journal of Dairy Science, v. 29, n. 2, p. 79-82, 1976.

[16] ZAMBIAZI, C. Oxidation reactions of vegetable oils and fats. Boletim SBCTA, v. 33, n. 1, p. 1-7, jan./jun. 1999. 